\title{
Control Strategies for a Dual AC Motor Pump System in Aircraft Hydraulic Power Packages
}

\author{
Nils Trochelmann and Frank Thielecke \\ Hamburg University of Technology, Institute of Aircraft Systems Engineering, Hamburg, Germany \\ E-mail: mailto:nils.trochelmann@tuhh.de; frank.thielecke@tuhh.de
}

\begin{abstract}
Hydraulic power packages (HPP) integrate electric motor-driven pumps (EMP) and hydraulic equipment to supply (on demand) hydraulic power to specific functions. To achieve a high operational availability two redundant EMPs are installed per HPP. To produce maximum output power, the EMPs need to operate in parallel. In the first part of this paper a baseline pressure control strategy is developed that enables the parallel operating mode. It has to cover the performance requirements and it is crucial to avoid stability issues of today's aircraft multi-pump hydraulic systems, induced by slightly differing pump characteristics. A central pressure controller, which calculates the total (cumulated) control effort and allocates it evenly to both EMPs, is selected as the basic controller structure. A loopshaping approach, where the requirements are mapped on target loop shapes, is applied. The control design is verified by nonlinear simulation and by experiments using representative aircraft prototype EMPs. Their slightly differing characteristics are utilized for an implicit proof of robustness. The second part of this paper makes use of the low utilization of the EMPs during most parts of the flight to achieve secondary objectives (efficiency, dynamic performance) and to improve the handling of operational constraints (e.g. electric input power limit). A model predictive control allocation (MPCA) algorithm flexibly allocates the total control effort to the EMP units. The allocation is derived from the solution of an optimization problem with the operational limits as constraints. Non-linear simulations of two exemplary scenarios show that the MPCA algorithm minimizes power losses by increasing the utilization of the more efficient unit. In addition, the MPCA algorithm improves the dynamic performance in case of an assymetric performance degradation by prioritizing the unit with better performance capability. The potential for improvement increases with the difference between the units and therefore offers advantages in dynamic allocation in particular for fault-tolerant operation.
\end{abstract}

Keywords: Hydraulic Power Package, Electric Motor-Driven Pump, (Model Predictive) Control Allocation, Parallel Pump Operation

\section{Introduction}

The electrification of the aircraft secondary power systems is a major approach to increase future aircraft efficiency, reliability, and to reduce operating cost. Under the guiding concept of More Electric Aircraft (MEA) different electrical system architectures have been investigated since the 1980s [1]. However, electric systems require electric actuation concepts. The main candidates are electro-mechanical actuators (EMA) and electro-hydrostatic actuators (EHA). Both still have issues that inhibit their frontline operation. For EMAs these are in particular prediction of jam-probability in safety critical functions, jam-tolerant control, high weigth and complexity in case of large power applications (like landing gear actuation). For EHAs pump reliability and heat rejection are the main concerns. In this situation, electro-hydraulic systems that supply hydraulic power from electric-motor-driven pumps (EMP) to conventional hydraulic actuators are a promising alternative. 


\subsection{Decentralized Electro-Hydraulic System Architectures}

In particular, decentralized architectures, as shown in fig. 1, are considered. They replace the central hydraulic system by a number of smaller zonal systems. Hydraulic power is generated by hydraulic power packages (HPP) and routed to the consumers of a specific zone through a local pipe network. This architecture eliminates the maintenance intensive central pipe system and allows proven and jam-free hydraulic actuation technology with high power density to be retained.

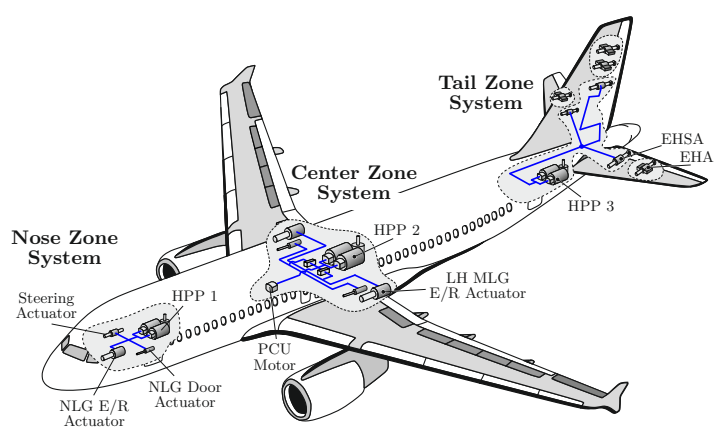

Figure 1: Decentralized Electro-Hydraulic System Architecture of a More Electric Aircraft

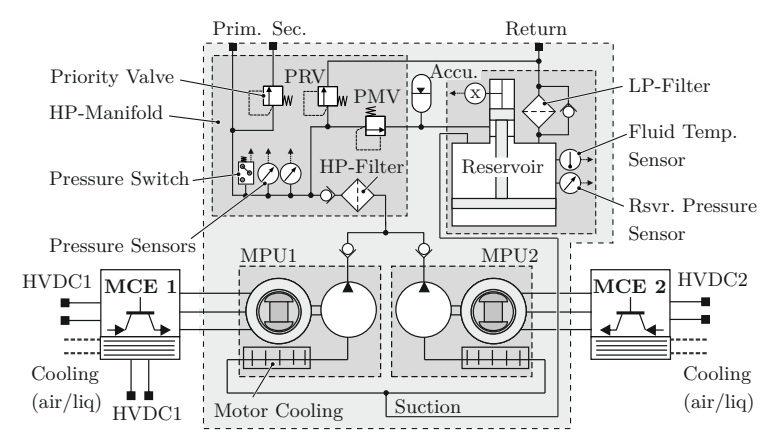

Figure 2: Exemplary Hydraulic Power Package Schematic

\subsection{Hydraulic Power Package}

The HPP is a key technology of new electro-hydraulic architectures. It integrates the EMP(s) and the hydraulic system equipment (accumulator, valves, filters, reservoir, sensors, etc.) in a compact easy-to-replace module. HPPs can be pre-assembled and pre-tested which permits quicker installation and cost savings. The HPP hydraulic schematic is illustrated in fig. 2. Two redundant EMPs are necessary to ensure the availability target [2]. For industrial purposes both EMPs are the same size. Check valves downstream of each pump avoid backflow to an inactive unit. For mass reduction, the EMPs are not sized for the maximum flow demand each. The EMPs are of type speed variable fixed displacement (VSFD), comprising a speed controlled permanent magnet synchronous motor (PMSM) and a fixed displacement pump. Electric power is modulated by the motor control electronics (MCE). The MCEs have a communication interface to the aircraft's avionic system.

For feedback pressure control and monitoring two redundant pressure sensors are installed. Pressure control is supported by a system accumulator covering transient flow peak demands that exceed the quasi-static maximum flow demand. The fluid is stored in a self-pressurizing bootstrap reservoir (pressurized air from the pneumatic system may not be available in the considered MEA scenarios [1]). A pressure maintaining valve (PMV) keeps a minimum reservoir pressure during pump start-up.

\subsection{Motivation and Objectives}

The HPP has to operate both EMPs in parallel to provide maximum hydraulic output power. Parallel pump operations can lead to undesired oscillations, e.g. triggered by (slightly) differing pump characteristics. A robust controller is developed that overcomes this issue and enables the stable parallel operation (duplex mode). The development steps concept synthesis, control, design, and experimental verification are presented.

Maximum output power is needed only for a short time during a typical flight mission, so that the dual EMP system is oversized most of the time. This opens up the potential to include additional/secondary goals, like minimization of power losses, and to handle operational constraints (e.g. power limitations) through dynamic control allocation of the two pump speeds, when the maximum output power is not requested. The development of a dedicated optimized control algorithm is the second objective of this contribution. First, chapter 2 gives an overview of existing multi-pump control concepts.

\section{Aircraft Multi-Pump Systems}

Many aircraft hydraulic systems operate multiple pumps, e.g. to increase availability or dissimilarity of hydraulic power sources. Mostly inline piston pumps with variable displacement and hydro-mechanical pressure compensators are used. For example, the AIRBUS A380 runs four engine driven pumps (EDP) per hydraulic system (a comprehensive overview is provided in [3]). Systems with multiple EMPs, like the HPP, are less common. One 
example is the BOEING 787 center system with two parallel EMPs. Though, the EMPs are normally not operated in parallel. The second EMP is activated only during phases of higher demand and/or to balance usage [4], [5].

Experiences with with parallel operations of pumps with hydro-mechanical compensation and associated findings are described in [6]:

- Pump-Flow-Fight: Pumps with slighty different characteristics attempt to dominate each other. This results in flow-fighting (crosstalk) and pressure oscillation. Pump-flow-fighting can be mitigated by different check valve pre-loadings or pressure compensator settings.

- Resonance: The superposition of pump pulsations can lead to resonances in the delivery line. This is avoided by (slightly) different pump speed settings.

- Instability: Instability due to a very low fluid volume can occur when a check valves is closed. This issue is more severe for pumps with low internal leakage (like the compensated IGP) and thus lower damping. Either compromising the compensator design and/or a redesign of the plant (e.g. increasing pump internal leakage, different check valve installation location) is necessary.

- Blocked Pump: In the above situations one pump could be blocked by the other and produce very low output flow. This leads to high internal pump temperatures and undesired, very low pump speeds in case of a speed variable pump.

The state of the art control solutions for multiple pump systems are limited to hydro-mechanical approaches. Electronically controlled pumps are not yet being used in commercial aircraft hydraulic systems [7]. A fighter aircraft hydraulic system with two parallel variable displacement pumps and electronic control is considered in [8]. The authors, however, do not detail the dual-pump control concept. A parallel configuration of two speed variable EMPs, which is very similar to the present application, is used for pump controlled landing gear actuation [9] and [10]. A closed loop pressure control with a dedicated pressure controller for each EMP is proposed. It should be mentioned that the pressure is sensed between check valve and pump so that the point of regulation is located directly at the pump's high pressure port, similar to a hydro-mechanical compensator. This arrangement is prone to the stability issues discussed above.

Multi-pump systems can also be found outside aerospace. In [11] the problem of an asymmetric loading of the pumps with hydro-mechanical compensators in a vessel hydraulic system is addressed. Electronic control concepts for pump controlled cylinders using multiple speed variable pumps, e.g. for moulding machines, can be found in [12], [13], [14]. These approaches cannot be transferred directly, because the EMPs are not redundant but control different process parameters (e.g. cylincer position, cylinder chamber pressure). To avoid inefficient part load phases dual-pump configurations are investigated that replace a single main pump by two smaller variable displacement pumps [15] or by a smaller primary pump and a VSFD EMP (operated in open loop) [16]. Last, energy efficient control strategies of parallel speed variable pump systems parallel centrifugal pumping systems were investigated in [17].

\section{Reference System}

The HPP concept from fig. 2 is emulated by the arrangement of two parallel EMPs. This is the reference system for the control development and verification in this paper. The test rig set up and the corresponding non-linear model are detailed below.

\subsection{Test Rig}

The test rig set up of the dual EMP system is depicted in fig. 3. Two aircraft prototype motor pump units (MPU) are supplied by industrial MCEs (Siemens SINAMICS). The combination of a MPU and MCE represents an EMP. The test rig hydraulic circuit includes the required utilities like accumulators, filters, pressure relief valves, and reservoirs. The consumers are emulated by a number of load valves (servo valves, flow control valves). The control and monitoring functions run on a dSPACE realtime platform, which basically provides the control inputs to the MCEs and valves, resp. reads the sensor signals.

The MPUs are nominally identical but their characteristics are slightly different due to different wear:

- The volumetric efficiency of pump 1 is slightly degraded. Its maximum output flow at nominal speed is only $431 / \mathrm{min}$ and lower than that of pump $2(451 / \mathrm{min})$.

- Compared to pump 2, the hydro-mechanical losses of pump 1 are increased by ca. $10 \%$ 


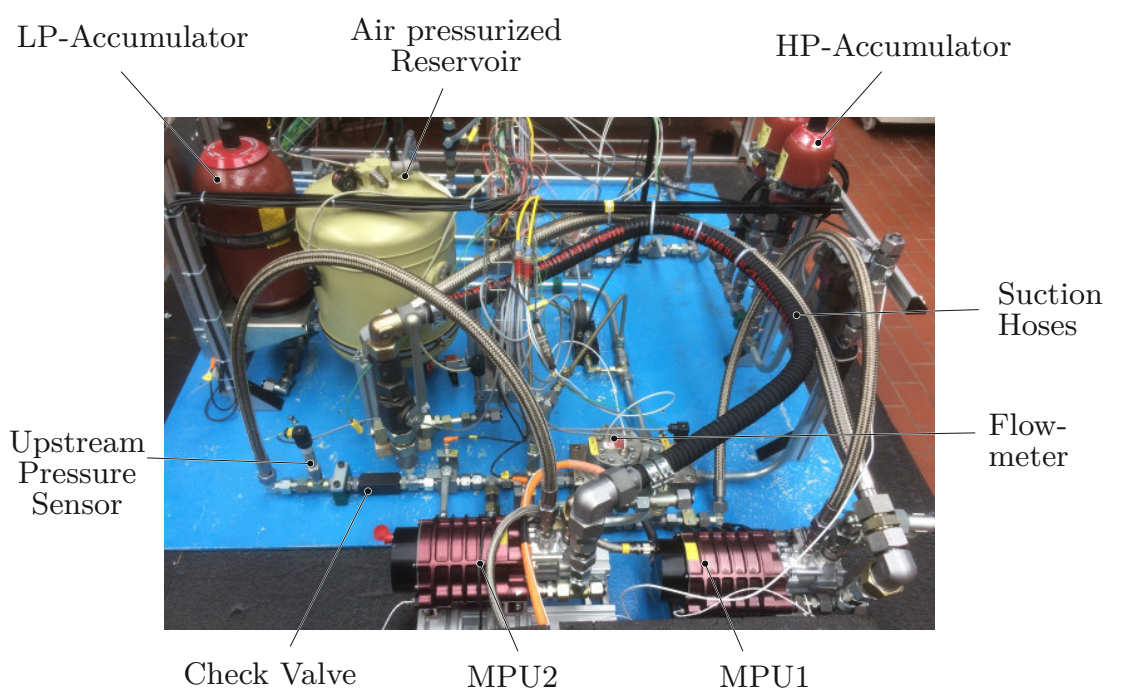

Figure 3: Experimental Dual EMP System Set Up

- Due to the power limitation of MCE 1, MPU1 maximum speed (95001/min) and current $95 \mathrm{~A}$ are below the nominal limits $(103501 / \mathrm{min}, 105 \mathrm{~A})$.

Notice that a comparison of the MPU power losses is illustrated in fig. 13.

The hydraulic integration of the pumps in the test rig follows the design of the HPP in fig. 2. The installation of check valves avoids mutual interferences. Pressure is measured upstream and downstream of the check valves. So, both sensor locations are available for regulation. The (combined pump) flow is measured downstream of the flow summing point and upstream of the accumulator. The suction line is splitted. The hydraulic jacket coolings are integrated in the separate line sections.

\subsection{Nonlinear Model}

A block diagram of the non-linear system model is illustrated in fig. 4. It comprises the two non-linear VSFD EMP models and a lumped system representation. The hydraulic consumers are represented by a load flow demand $Q_{L}$. The model (plant) inputs are the voltage set points $u_{q, c, i}$. The output is the pressure in the downstream system $p$. The non-linear plant model is implemented in MATLAB/SIMULINK.

The VSFD EMP model follows the approach detailed in [18] which achieved high accuracy. In this model the MCE dynamics, i.e. the time to set the commanded voltage, is modeled by a first order approximation of [19]. The PMSM is modeled as typical two phase system applying the park transformation [20]. The $\mathrm{d}$ - and q-axis currents $i_{q}, i_{d}$ are calculated by the stator voltage equations. The resulting electromagnetic torque is obtained from resulting currents and the linked flux. The rotor speed $\omega_{m}$ is derived from the mechanical equation.

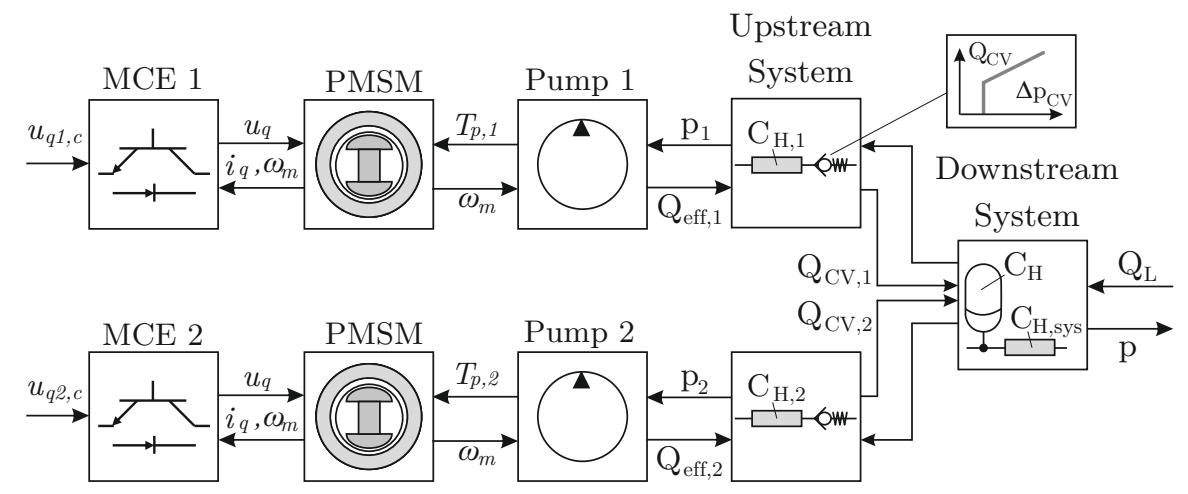

Figure 4: Non-Linear Duplex EMP Control Design Model

The pumps are modeled as combinations of ideal fixed displacement pumps and volumetric, resp. hydro-mechanical loss models, which are functions of speed, pressure, and fluid temperature, derived from measurements. The 
pumps output the effective flows $Q_{e f f, i}$ and require the drive torque $T_{p, i}$. The pipe segments between pump and check valves, referred to as the upstream system, are represented by lumped capacities $C_{H, i}$. The pressure build-up in the upstream capacities $C_{H, 1 / 2}$ is described by

$$
\dot{p}_{1 / 2}=\frac{Q_{e f f, 1 / 2}-Q_{C V, 1 / 2}}{C_{H, 1 / 2}}
$$

where $Q_{e f f, 1 / 2}$ are the effective pump output flows and $Q_{C V, 1 / 2}$ are the check valve flows. In the open position these are calculated by the orifice equation

$$
Q_{C V, 1 / 2}=B_{C V, 1 / 2} \cdot \sqrt{p_{1 / 2}-p_{s y s}}
$$

with the orifice coefficient $B_{C V, 1 / 2}$. The pressure build-up in the downstream system capacity, which is formed by the lumped accumulator and downstream pipe system capacities, yields

$$
\dot{p}_{s y s}=\frac{Q_{C V, 1}+Q_{C V, 2}-Q_{L}}{C_{H, s y s}},
$$

where $C_{H, s y s}$ is also a function of the pressure (compare [18]). Solving eq. (2) for $p_{1 / 2}$, inserting the result and (3) into eq. (1) yields the expression

$$
Q_{C V, 1 / 2}=Q_{e f f, 1 / 2} \cdot \frac{C_{s y s}}{C_{H, s y s}+C_{H, 1 / 2}}+\left(Q_{L}-Q_{C V, 2 / 1}\right) \cdot \frac{C_{H, 1 / 2}}{C_{H, 1 / 2}+C_{H, s y s}},
$$

which describes the cross-coupling between the two check valve flows. The coupling is mainly characterized by the fraction of the system and upstream capacities. In particular, the coupling is strong for large $C_{H, 1 / 2}$ which yields

$$
\frac{\partial Q_{C V, 1 / 2}}{\partial Q_{C V, 2 / 1}}=-\frac{C_{H, 1 / 2}}{C_{H, 1 / 2}+C_{H, s y s}} \approx-1
$$

The coupling is weak for large $C_{H, s y s}$, respectively. So, to reduce mutual interferences

- $C_{H, s y s}$ should be designed large in comparison to $C_{H, 1 / 2}$

- and the variations of $Q_{C V, 2 / 1}$ should be compensated by adjusting $Q_{e f f, 1 / 2}$, e.g. by a flow synchronization.

\section{Baseline Controller Synthesis}

This section develops a baseline pressure controller for the duplex operation mode. After the specification of the requirements and the selection of a suitable control structure, a linear design model is created. The subsequent design and tuning apply a loopshaping design method, presented in [18]. The control design is verified and validated by non-linear simulation and an experiment.

\subsection{Requirements Specification}

The main task of the pressure controller is to maintain the required supply pressure by tracking the reference pressure and reject disturbances with zero steady state error. The stability of the closed control loop shall be ensured by satisfying a maximum gain margin $G M \geq 6 \mathrm{~dB}$ and a minimum phase margin $P M \geq 60^{\circ}$. High frequency noise has to be blocked from entering the feedback loop. A general objective is to keep design simple and transparent for low complexity and certification effort.

Pressure control performance requirements are stated by SAE AS 595 [21]. In particular, the performance is specified by a pressure response to a load step from minimum to maximum flow demand and vice versa, as illustrated in fig. 5. It is characterized by the parameters of tab. 1. These are minimum and maximum pressures $p_{\min / \max }$, the response times $\left(t_{1}, t_{2}\right)$, and the settle time $t_{3}$. The response time specifies the time to transit from minimum to maximum output flow and vice versa. The settle time is the time for the pressure to settle back to the rated level. Note that there is no explicit requirement for $p_{\min }$, so it is assumed to be symmetric to $p_{\max }$.

The EMP speed must stay within the operating limits. The SAE AS 595 recommends to not exceed $115 \%$ of the rated speed as upper limit. In addition, a minimum speed limit of $5001 / \mathrm{min}$ (well above the physical limit) is applied, to be conservative. Further, to limit wear effects, acceleration shall not exceeed $\pm 50.000 \mathrm{rpm} / \mathrm{s}$. 
Table 1: Dynamic Requirements

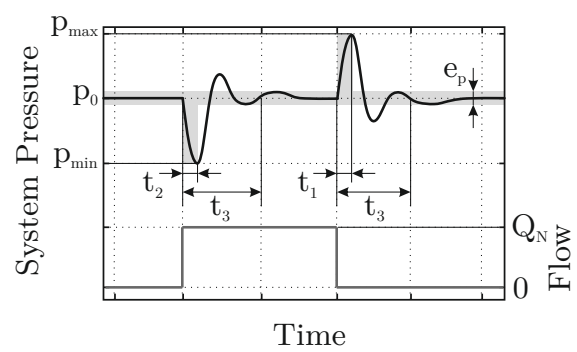

\begin{tabular}{clcc}
\hline Parameter & Description & Value & Unit \\
\hline$p_{0}$ & Nominal System Pressure & 206 & $\mathrm{bar}$ \\
$t_{1}$ & Response Time & 50 & $\mathrm{~ms}$ \\
$t_{2}$ & Response Time & 100 & $\mathrm{~ms}$ \\
$t_{3}$ & Settling Time & 1 & $\mathrm{~s}$ \\
$p_{\max }$ & Max. Transient Pressure & 257.5 & $\mathrm{bar}$ \\
$p_{\min }^{*}$ & Min. Transient Pressure & 154.5 & $\mathrm{bar}$ \\
\hline
\end{tabular}

Figure 5: Step Response

Last but not least, to avoid the issues of duplex operation mentioned in section 2, the control shall offer robustness against dissimilar EMP characteristic.

\subsection{Pressure Control Concept Selection}

A cascaded control, in which the speed control loops and their subordinate current controllers have already been suitably designed, is taken as basis. Then, the task of the pressure controller is to calculate the required motor speeds $\omega_{c, 1 / 2}$, for which different concepts are possible by varying the following properties:

- The point of regulation is defined by the location of the pressure sensor. The pressure sensors can be either installed upstream or downstream of the check valves. It has a significant impact on the controller performance and structure.

- The hierarchy defines the EMP role. In the related field of electronic synchronizing distributed electric drives ( [22], [23], [24]) the following concepts are distinguised:

- Peer-to-peer (P2P): All (electric) drives have (almost) equal authority. Decentralized and centralized controller structures are possible.

- Master/Slave: One leading drive represents the master, which follows the commands of a supervisory level controller. The other drives (slaves) track the reference signal of the master.

The resulting concepts are illustrated in fig. 6. Concepts I-III are discarded because the upstream pressure sensor location was found to be disadvantagous for stability and performance, mainly due to the non-linear behaviour of the check valves. The downstream concepts IV-VI avoid these issues in principle since opening and closing of the check valves has low impact on the downstream pressure. In addition, the downstream sensor arrangement is advantagegous for disparity monitoring.

In concept VI the slave EMP tracks the master EMP speed, so it adapts to the master EMP automatically without complex monitoring. Yet, this approach decreases the overall performance because the tracking is non-ideally fast. Another drawback is that the slave EMP does not compensate for a degradation of the master EMP. The M/S concept is also discarded.

The remaining P2P concepts IV and V have different controller structures. In concept IV a centralized pressure controller commands a cumulated total speed which is allocated to both EMPs. In concept V the speed commands are calculated by two decentralized pressure controllers, which receive the same reference and feedback. The main advantage of the central controller (concept IV) is the simple (re-)configuration between simplex/duplex mode and the possibility to flexibly allocate the total effort. That is why concept IV is chosen.

\subsection{Design and Tuning}

The detailed design of the selected pressure control concept is illustrated in fig. 7. Based on the control error $e_{p}=p_{c}-p$ the pressure controller $C_{p}$ calculates a total control effort $v$, which represents the total required speed. The EMP speed commands $\omega_{c, i}$ are determined by the allocation algorithm (allocator) via

$$
\left[\omega_{c, 1} \omega_{c, 2}\right]^{T}=\left[\alpha_{1} \alpha_{2}\right]^{T} \cdot v \quad,
$$




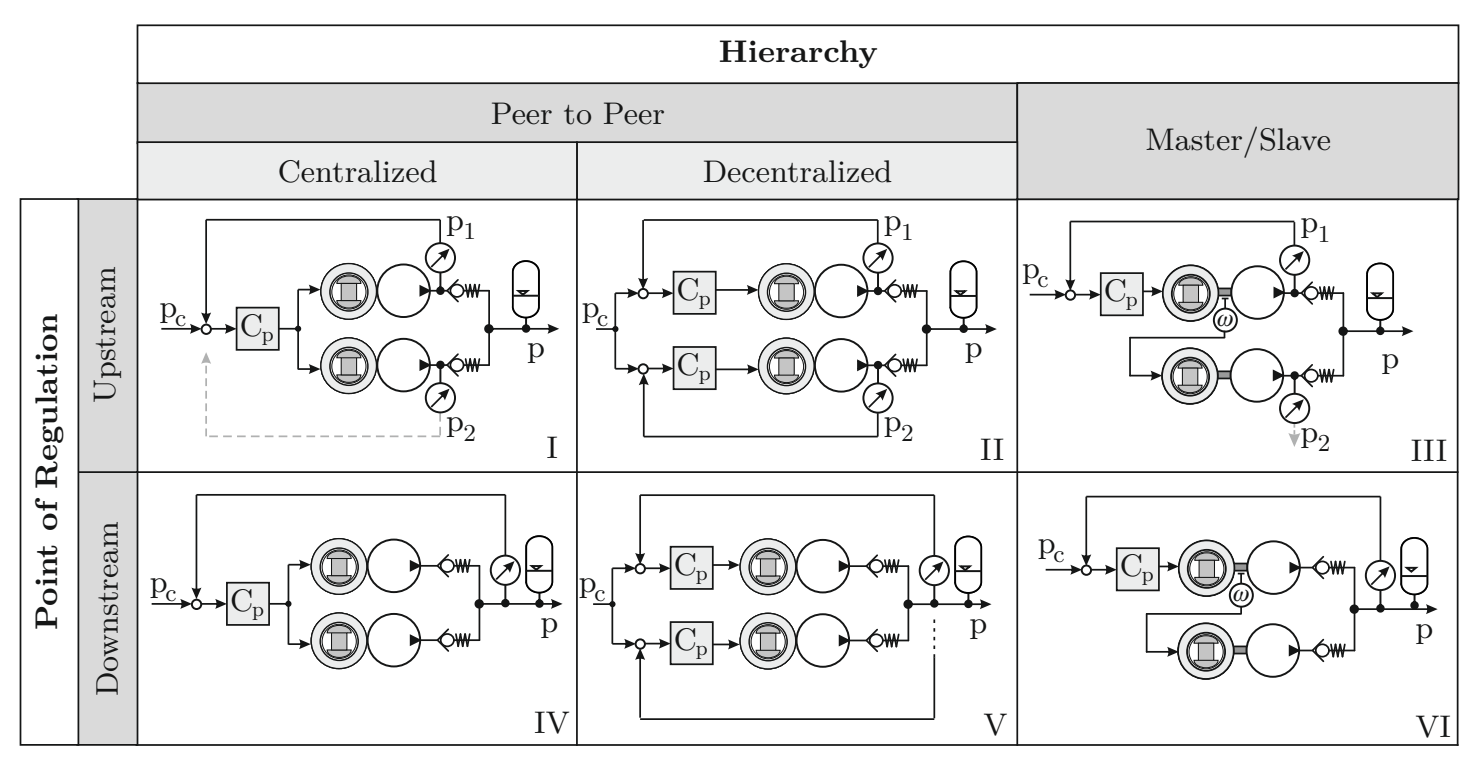

Figure 6: Classification of Control Concepts

introducing the allocation factors $\alpha_{1,2}$. In the nominal case, which is considered here, the effort is allocated evenly $\alpha_{1}=\alpha_{2}=0.5$. Note, that simplex operation is achieved by simply setting $\alpha_{1 / 2}=1$ and $\alpha_{2 / 1}=0$. In the following a linear design model is derived.

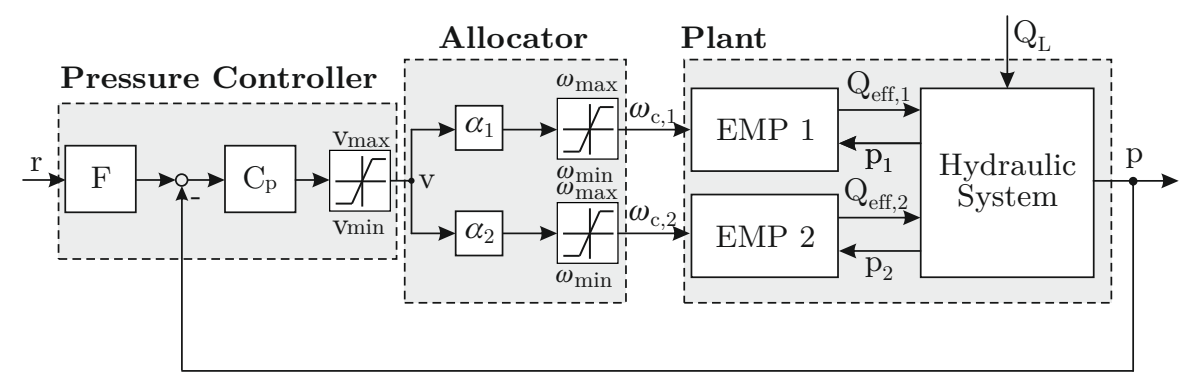

Figure 7: Detailed Design of the Dual EMP Baseline Control Concept

\subsubsection{Linear Design Model}

The plant block diagram representation is illustrated in fig. 8. It comprises the closed speed control loops, which are represented by second order transfer functions $T_{\omega, c l}$. The pump transfer function is $G_{p, i}$, where $k_{l e, \omega, i}$ denotes the linear coefficient of speed-dependent leakage. The concatenate $T_{\omega, c l} \cdot G_{p, i}=G_{M P U, i}$ yields the MPU transfer function. The check valves and the upstream capacities are neglected. This approximates the open check valve and is a reasonable assumption for the nominal operation. The system pressure build-up is modeled by the system transfer function $G_{s y s}$, where $k_{l e, p, i}$ represents the pressure-depending internal pump leakage and $C_{s y s}$ is constant. Note that $G_{s y s}$ is identical to the disturbance transfer function $P_{d}$, which describes the relation between disturbance $Q_{L}$ and output $p$.

For the nominal system it is assumed that both MPUs are identical, introducing $G_{M P U}=G_{M P U, 1}=G_{M P U, 2}$. This simplifies the plant to a SISO system shown in fig. 9.

For the controller design the allocation factors are set to $\alpha_{i}=0.5$, which yields the loopgain to be

$$
L(s)=C_{p}(s) \cdot T_{\omega, c l} \cdot G_{p} \cdot G_{s y s}=C_{p}(s) \cdot P(s)
$$

where $P(s)$ is the SISO system plant.

\subsubsection{Loopshaping Design and Tuning}

The simplification to a SISO system enables the application of the loopshaping method proposed in [18]. It facilitates a systematic design and a low order controller. The principal idea of classical loop-shaping is to shape 


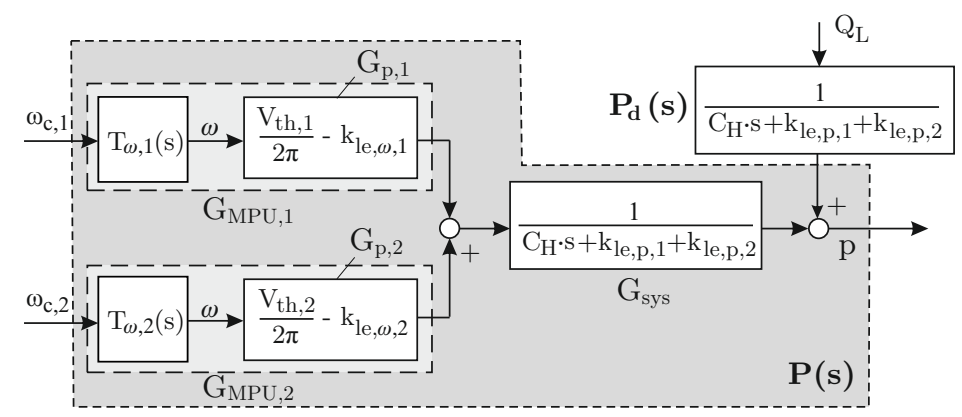

Figure 8: Block Diagram of the Plant

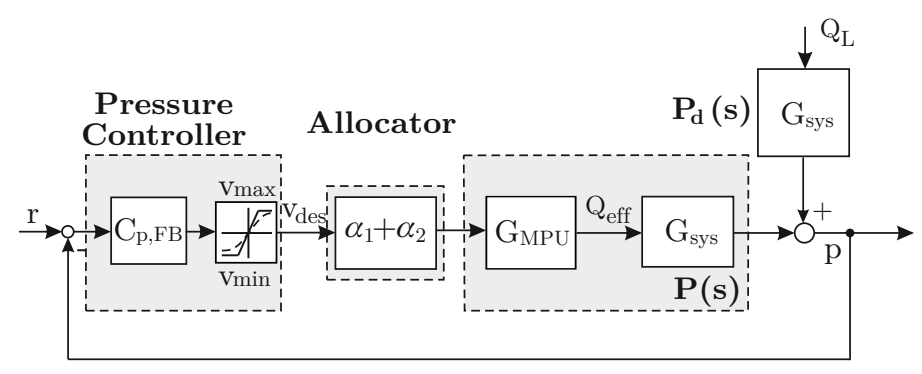

Figure 9: Simplified Closed Loop System in SISO Configuration

the open loop transfer $L(s)$, specifically its magnitude to a defined (target) loop shape. In parallel, stability margins can be evaluated from the phase. In the following the performance requirements are mapped onto target loop shapes.

Specification of Target Loopshapes The performance requirements and constraints have to be mapped onto specific closed loop transfer functions. For this purpose the following set of closed loop transfer functions is introduced:

$$
\begin{gathered}
S(s)=\frac{1}{1+L(s)}, \quad T(s)=\frac{L(s)}{1+L(s)}, \quad S P_{d}(s)=\frac{P_{d}(s)}{1+L(s)} \\
C S(s)=\frac{C(s)}{1+P(s) \cdot C(s)}, \quad C S P_{d}(s)=\frac{C(s) \cdot P_{d}(s)}{1+P(s) \cdot C(s)}
\end{gathered}
$$

The sensitivity $S$ characterizes the impact of a disturbance, acting on the output of the plant. The complementary sensitivity $T$ describes the closed loop transfer function from reference to output. Ideally $S(s)$ shall be small for a small control error. At the same time $T(s)$ shall be small for low noise sensitivity [25], which is a typical design conflict. Further, $S P_{d}(s)$ denotes the disturbance sensitivity transfer function, which characterizes the transfer of a (load) disturbance on the output. The disturbance rejection requirements, defined in sec. 4.1, are mapped onto a target loop shape for $P_{d} S(s)$, as proposed in [18]. In general, $S P_{d}(s)$ should be low at low frequencies, to limit the impact of disturbances. It should also be low at high frequencies to attenuate noise. The maximum magnitude corresponds to the overshoot requirement, whereas its frequency - in good approximation - corresponds to the bandwidth. The controller sensitivity $C S(s)$ describes the transfer from the reference or the noise noise input to the controller output $u$. Its magnitude should be high at low frequencies for a responsive controller but low at high frequencies to attenuate the noise. The controller disturbance sensitivity $\operatorname{CSP}_{d}(s)$ characterizes the impact of a disturbance on the control signal. An upper bound on $\operatorname{CSP}_{d}(s)$ limits the maximum speed command due to the maximum disturbance. Moreover, an upper bound on $\operatorname{CSP}_{d}(s) \cdot s$ limits the corresponding rate. The resulting target loop shapes $W_{i}$ (grey lines) are illustrated in fig. 10 (b).

Tuning Result The tuning is carried out using MATLAB's sisotool. Both, design targets and resulting loopshapes are illustrated in fig. 10. The controller is designed primarily to meet the stability and disturbance rejection 
performance requirement $\left(W_{S P_{d}(s)(s)}\right)$. As a result a PI control law is obtained to be a suitable choice, which matches most of target loopshapes.
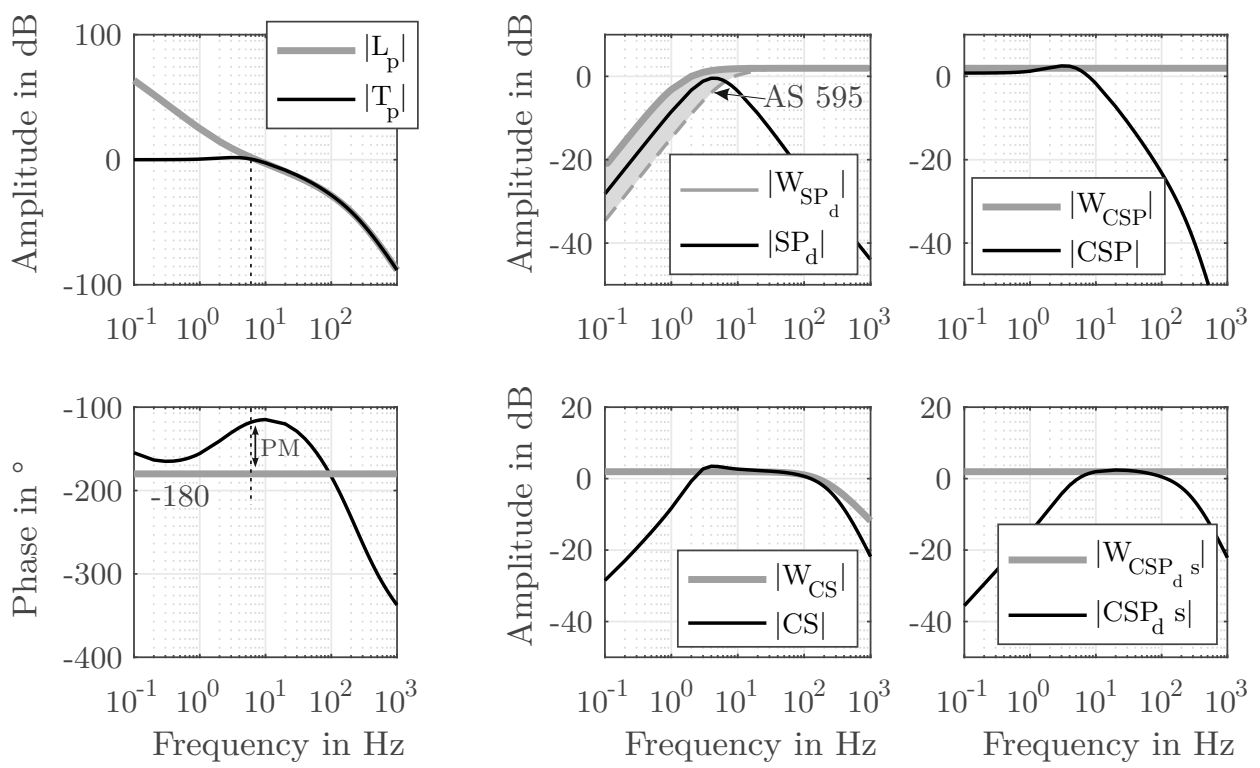

(a) Open Loop Frequency Response

(b) Closed Loop Frequency Responses

Figure 10: Target Loopshapes and Controller Tuning Results

The evaluation of the phase angle $\left(115^{\circ}\right)$ at the crossover frequency $\left(\omega_{L, 0}=8 \mathrm{rad} / \mathrm{s}\right)$ implies that the design has sufficient stability margin $\left(P M=65^{\circ}\right)$. In terms of performance the $S P_{d}(s)$ magnitude reveals that the overshoot requirement is satisfied, while the response time requirement cannot be met. The magnitudes of $\operatorname{CSP}_{d}(s)$ and $\operatorname{CSP}_{d}(s) \cdot s$ indicate that speed and rate limits are satisfied, so the system is at the design limit here.

\subsection{Design Verification}

The controller design is verified regarding stability and dynamic performance (disturbance rejection capability). Figure 11 compares the simulation of a load step with the nonlinear model to the experiment. Initially, the system is compensating the static flow demand of $101 / \mathrm{min}$. Here, the most essential finding is that the pump speeds are synchronized well and that no oscillations are present. After one second a load step to 801/min (maximum considered load) is applied. The controller compensates the load by increasing the speed command. Both EMPs accelerate at their maximum rate. The details reveal that the EMP 1 acceleration is slower (by ca. $10000(1 / \mathrm{min}) / \mathrm{s}$ ) due to the limited performance. Yet, no fighting or instability is observed. This is an implicit proof of the robustness against dissimilarities. During the load step back to 101/min both EMPs have the same peformance, because of the aiding hydraulic load torque. The minimum pressure (170 bar) and the maximum pressure (250 bar) stay within the limits. This shows that the controller satisfies the performance requirements and avoids the issues of multi-pump systems with hydro-mechanical compensation. Last but not least, simulation and experiment show an acceptable match. So, the model of the duplex system appears suitable for further model based investigation.

\section{Flexible and Optimal Control Allocation}

During flight maximum hydraulic power is needed only temporarily. This means the dual EMP system is oversized and thus overdetermined most of the time. During these phases the low utilization opens up potential to include secondary objectives (efficiency, dynamics) and/or to handle varying operational constraints (e.g. power limitations of electric supply system) through dynamic allocation. This section develops and investigates a flexible control strategy based on a model predictive control scheme.

\subsection{Potentials of the Low Utilization}

Flexible dynamic allocation is possible when the available total flow exceeds the demand $Q_{M P U, 1, \max }+Q_{M P U, 2, \max }>$ $Q_{L}$ and neither MPU is saturated $\left(Q_{M P U, i}<Q_{M P U, i, \max }\right)$. Then, it is possible to satisfy secondary objectives and/or to handle specific operational constraints. In this paper the following objectives and constraints are considered. 

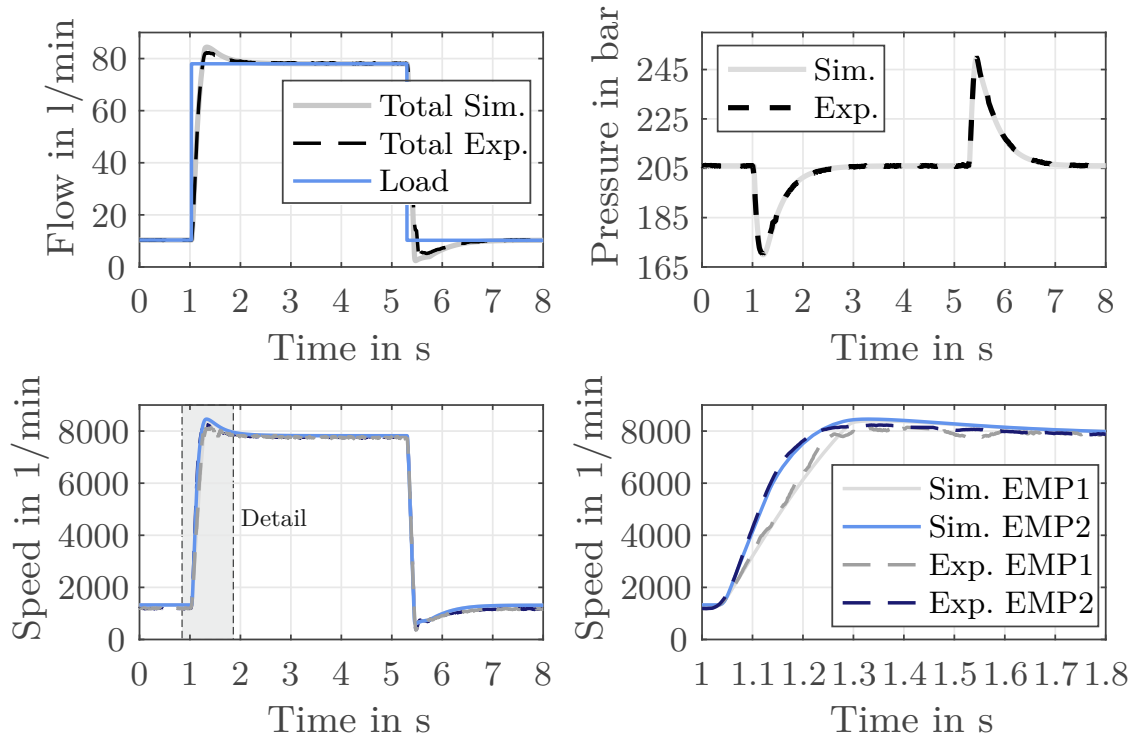

Figure 11: Control Design Verification and Model Validation

\subsubsection{Secondary Objectives}

In addition to the primary control objective, i.e. to ensure the required output power, the controller shall consider the following secondary objectives:

- Minimization of power losses: The requested hydraulic output power is provided at maximum efficiency, i.e. with minimum power losses.

- Maximization of dynamic performance: A useful measure of the dynamics is the gradient $d Q / d t$. Since $Q_{i} \sim \omega_{i}$, in this paper maximizing $d v / d t$ is used as a suitable approximation (neglecting the impact of different internal pump leakage).

The approach could be extended to include additional objectives, for example minimization of operating noise or reducing wear, e.g. by balancing the usage and by penalizing fast accelerations.

\subsubsection{Operational Constraints}

The operation of the HPP is subject to numerous boundary conditions, which are imposed by the inherent monitoring functions and by adjacent systems. A controller with flexible allocation can improve the restriction handling resp. enable it at all. This is illustrated by the following examples:

- Health and Usage Monitoring (HUMS): The HUMS monitors the health condition and usage of the system. In order to compensate for the degradation of a component, the HUMS could require the controller to adapt the EMP operating limits or to balance their usage by prioritizing one unit. Then, certain operating points (e.g. very high or low speed or certain frequency bands) may have to be avoided.

- Failure Monitoring: This function detects failures in the hydraulic system and triggers a dedicated reaction. For example, a detected reservoir low pressure condition could require to limit the speed or rate to prevent from cavitation.

- Electric Power Management: The electric power system is an important adjacent system. Specifically, the electric power management may impose limitations on the available HPP input power. For example, in a emergency a limited amount of power has to be shared among different (electrical) functions. Then, the voltage level and/or current available for the HPP may be reduced. A reduced voltage in turn limits the maximum speed and a reduced current limits the maximum torque (and consequently dynamic performance).

\subsection{Concept Definition}

Based on the above considerations the optimized control concept shall offer the following capabilities: optimization of the operating behavior with respect to secondary objectives, handle varying operating constraints. This section 
selects and defines a suitable control strategy. For that, the tasks of (higher-level) pressure control and control allocation, shall be separated, in order to retain the proven pressure controller. This is expected to be advantageous in terms of transparency and acceptance, as the system can recover to the baseline control any time by fixing the allocation. Then, the control task narrows down to a typical control allocation problem.

A comprehensive overview of different control allocation methods is given in [26]. In this paper a model predictive controller (MPC) is used to solve the allocation problem, which is referred to as model predictive control allocation (MPCA). This approach calculates the control allocation by optimization of the predicted behaviour over a finite horizon. The main advantage of MPCA over simple algorithms - like direct allocation or daisy chaining - is the ability to explicitly take into account the actuator (here EMP) dynamics as higher order models and the systematic handling of actuator limits. MPCA is widely used in process industry [26], but also applied for braking torque blending in vehicle control [27] or in flight control [28]. For usage of MPC to optimize hydraulic process controls refer in particular to WILLKOMM [29]. An approach to reduce the computational effort (by structured MPC) is presented for a cylinder drive in [30].

Figure 12 illustrates the proposed structure of the HPP control including the MPCA. The (high-level) pressure controller computes a required total control effort $v$. The main objective of the MPCA algorithm is to ensure that this control effort is produced (jointly by all EMPs) at all times, while meeting the secondary objectives and satisfying the constraints.

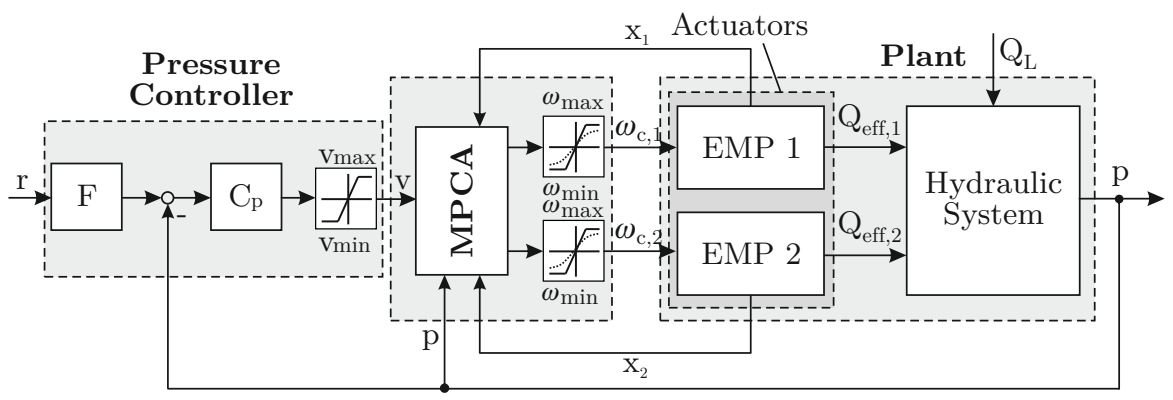

Figure 12: HPP Control Concept including MPCA

\subsection{Implementation and Optimization Problem}

The MPCA implementation used is based on the MPC formulation in [31]. Specifically, the objective of the MPC is to find a finite horizon of $N_{c}$ (control horizon) control inputs $u_{k}$ that minimize an objective function and satisfy the constraints over the prediction horizon $N_{p}$. More specifically, here $u_{k}=\omega_{c_{k}}=\left[\omega_{c, 1, k} ; \omega_{c, 2, k}\right]$ is a vector that contains the two control commands, which are the decision variables of the optimization problem. The objective function is

$$
J=\min \left\{\lambda_{v} \cdot \sum_{k=1}^{N_{p}}\left(y_{r e f, k}-y_{k}\right)^{2}+\lambda_{\Delta u_{k}} \cdot \sum_{k=0}^{N_{c}-1} \Delta u_{k}^{2}+\lambda_{P_{V}} \cdot \sum_{k=1}^{N_{p}} P_{V}\left(x_{m, k}, \Delta u_{k}\right)\right\}
$$

It is formed by different terms to account for different objectives, which can be blended by the weighting factors $\lambda_{i}$. The first term minimizes the error between the set point $y_{r e f}=v$ and the predicted output $y=\omega_{1}+\omega_{2}$. The second term penalizes the control effort. The third term minimizes the total power losses $P_{V}=f\left(\omega_{1}, \omega_{2}, p, \vartheta_{f l}\right)$ to optimize the efficiency. The power losses are a function of speed, pressure, and fluid temperature, which are based on experimental measurements, as is detailed in sec. 5.4. The optimization is subject to the following constraints

$$
u_{i, \min } \leq u_{i} \leq u_{i, \max } \quad, \quad \Delta u_{i, \min } \leq \Delta u_{i} \leq \Delta u_{i, \max }
$$

which allows physical limitations of the system to be incorporated. In this paper the speed limits and rate (acceleration) limits are considered as constraints because current and voltage limits are satisfied by the subordinate control loops. Note that current and voltage limits can be formulated implicitly by speed and rate limits, too. The constraints are provided by the supervisory control level. The prediction of system states $x_{k}$ and outputs $y_{k}$ uses a discrete time state space representation 


$$
\begin{aligned}
x_{k+1} & =A \cdot x_{k}+B \cdot \Delta u_{k} \\
y_{k} & =C \cdot x_{k}
\end{aligned}
$$

which includes not only the EMPs but also the system, because the pressure retroacts on the motors via the pump torque. Notice that the input is $\Delta u_{k}=u_{k}-u_{k-1}$ and $A, B, C$ represent an augmented model with an integrator. The augmented state vector $x_{k}=\left[\Delta x_{m, k}^{T} y_{k}\right]^{T}$ is formed by the state increments $\Delta x_{m, k}=x_{m, k}-x_{m, k-1}$ (motor currents, speeds, and pressure) and the output $y_{k}$. The objective function is solved at each time step, to obtain the optimal sequence of $\Delta u$, resp. of $u$. Only the first element of $\Delta u$ is commanded to the motors. This procedure is repeated at each sample instant (receding horizon control).

\subsection{Evaluation}

The performance and potential of the MPCA strategy are evaluated in comparison to the baseline concept, using efficiency and dynamic performance as examples.

\subsubsection{Energy Efficiency}

The efficiency optimization requires to minimize the power losses, while ensuring the allocation of the total speed. To achieve this objective the power loss term is activated in the objective function by setting $\lambda_{v}=\lambda_{P_{V}}=1$. In addition, including the control effort in the objective function $\left(\lambda_{\Delta u_{k}}=1\right)$ is necessary to avoid high frequency variations of the control output. The power loss function is evaluated exemplarily for 206 bar and $30^{\circ} \mathrm{C}$ fluid temperature in fig. 13 over $v$ and $\omega_{1}$, where implicitly $\omega_{2}=v-\omega_{1}$. An offline optimization yields the optimal $\omega_{1}$ for each $v$. It shows a priorization of unit two, in particular at higher speeds, which is in line with the increasing delta between the power losses in fig. 13 (b).
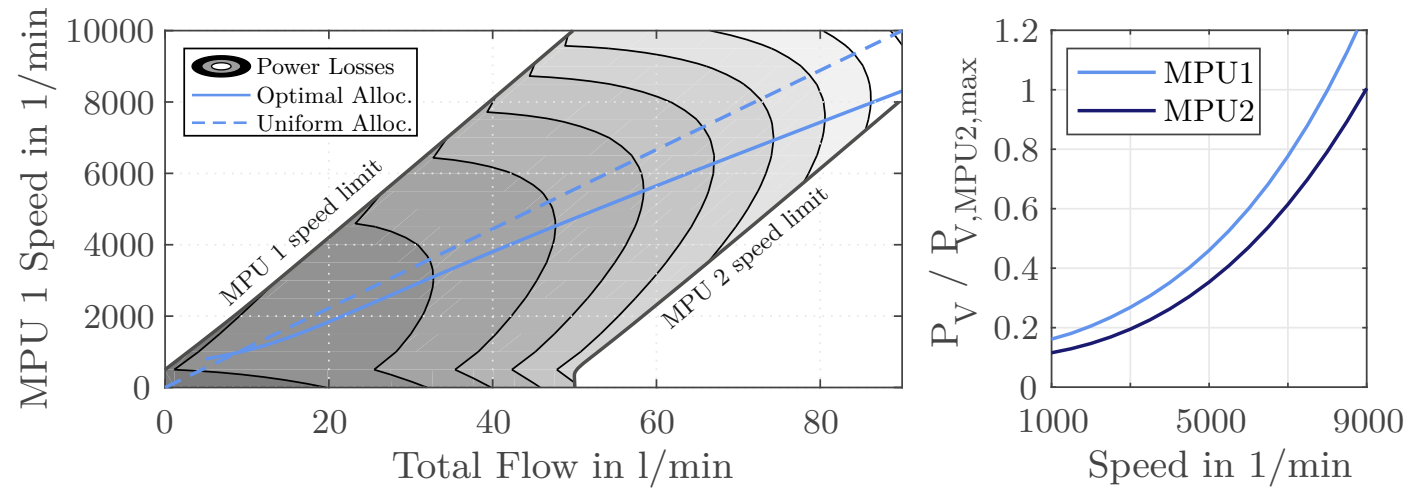

Figure 13: Comparison of Power Losses and Static Offline Efficiency Optimization

For the evaluation of the efficiency optimization a test scenario is defined, which includes different load conditions of a potential HPP operating profile. The pressure controller maintains a constant system pressure of $206 \mathrm{bar}$. The nominal limitations are applied as constraints. The simulation results for the baseline and the MPCA control concept are compared in fig. 14. From the top to the bottom the plot depicts the consumer flow demand (load) and total pump output flow, system pressure, pump speeds, and the delta energy loss between MPCA and baseline. Comparing the pressure regulation quality, no dynamic performance differences are visible over the entire sequence. So, the MPCA approach does not lead to adverse system behaviour here, which is an essential prerequisite.

Comparing the speed allocation, the MPCA scheme makes slightly more use of the second (more efficient) unit at low speed. However, considering the energy loss, the potential savings are low here, because the increased loss at an increased speed appears to cancel out the savings at the less efficient unit. For low flows energy savings are possible by switching off the unit with poorer efficiency, which is not detailed here. At higher flow demands the situation is different. The more efficient EMP 2 is prioritized considerably (e.g. at the flow demand of 801/min the delta between the commanded speeds is about $10001 / \mathrm{min}$ ) as the different efficiencies have a greater impact here. During dynamic phases increased motor current, required for acceleration, causes additional losses in both units. So, both units are accelerated similarly. The dynamic loss fraction is however low compared to the static fraction. In conclusion, the MPCA approach enables the same dynamic performance while a slight improvement 
of the efficiency is possible. Qualitatively it can be expected, that the potential energy savings increase for greater differences in the efficiencies.
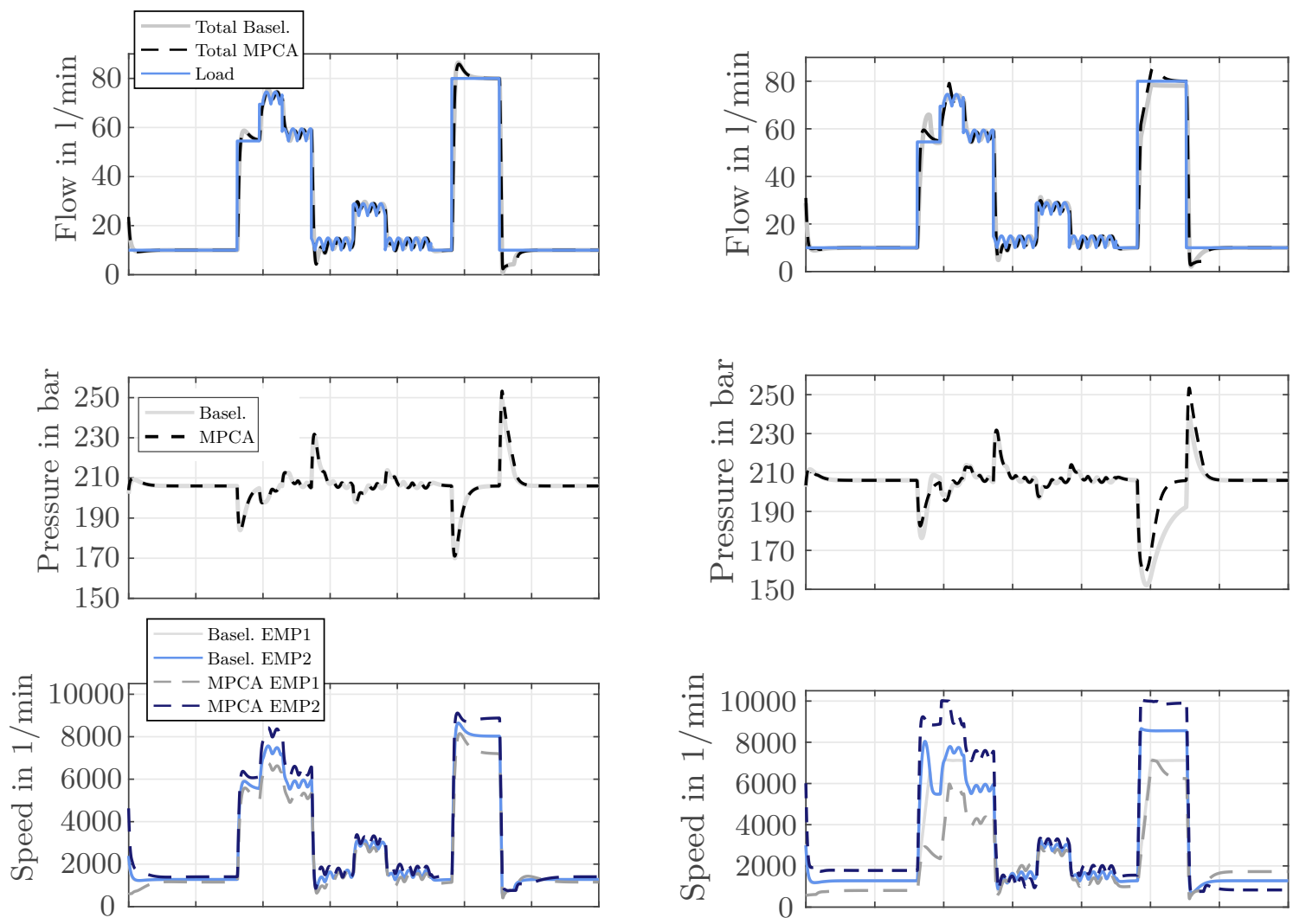

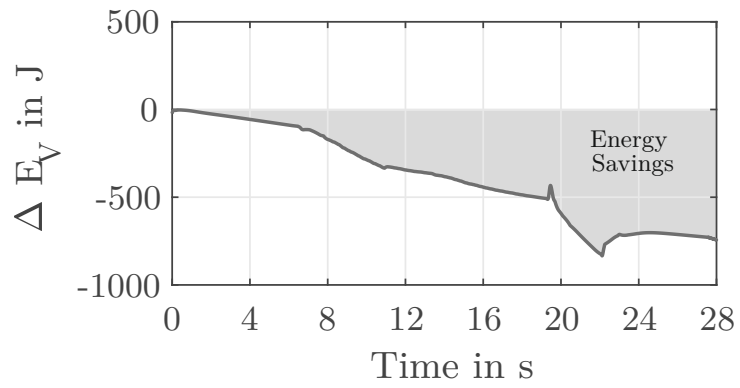

Figure 14: Result Efficiency

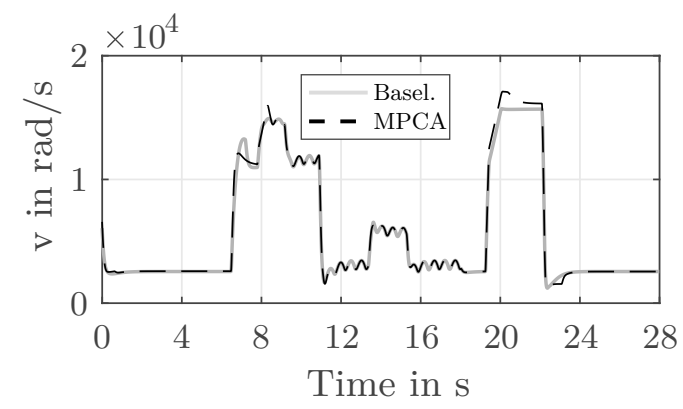

Figure 15: Result Dynamic

\subsubsection{Dynamic Performance}

According to the definition given above, the optimization of the dynamic performance is equivalent to eliminating the error between $v$ and $\omega_{1}+\omega_{2}$ as fast as possible. This objective is pursued by prioritizing the first term of eq. 10. Again, the second term is kept for damping, so that $\lambda_{v}=\lambda_{\Delta u_{k}}=1$ and $\lambda_{P_{V}}=0$ (no efficiency optimization).

In case of two units with identical dynamic performance the best overall dynamic performance is achieved by a uniform allocation. Then each single unit has to accelerate to a relative lower target speed. In this case there would be no optmization potential for the MPCA algorithm. The situation is different for two units with (asymetrically) degraded dynamic performance. Then, the MPCA algorithms can generate an advantage by prefering the better unit. This potential becomes apparent by considering a worst case scenario for MPU1:

- The HUMS detected a significant degradation of the hydraulic pump efficiency. Specifically, the friction torque is increased by $10 \%$.

- The electric power management requests to limit the EMP1 power consumption (e.g. in an emergency condition). Specifically, 
- the input voltage is reduced to $75 \%$,

- the maximum current is limited to $80 \%$.

The combination of lower maximum current and increased friction torque reduce the acceleration capability to $15 \%$ of the nominal capability. The reduced voltage level limits the maximum nominal speed and flow. Limited speed and rates of EMP 1 are taken into account by the MPCA by adapting $\Delta u_{i, \max }, u_{i, \max }$. The simulation result is illustrated and compared to the baseline in fig. 15.

The potential for improvement of the MPCA already becomes clear in the first load step. The MPCA algorithm allocates almost the entire speed to the non-degraded EMP 2. This partly compensates the degradation of EMP1, which improves the pressure control quality considerably (lower pressure undershoot, shorter settling time). During the subsequent load reduction no difference is observed, since negative acceleration is aided by the hydraulic pump torque.

The performance difference becomes also clear in the second load step. Here EMP 1 is driven into the (reduced) speed limit. The MPCA algorithm compensates the saturation by increased utilization of MPU2. This improves the pressure control quality. Again, there is no principle difference during load reduction. The baseline controller pressure overshoot is lower only because the pressure had not been fully recovered before the load step. In summary, the exemplary analysis showed that the MPCA algorithm is able to compensate for asymmetric characteristics, resp. operational constraints. This allows the system to maintain improved performance even in a degraded state.

\section{Conclusions and Outlook}

In the first part of this paper an electronic pressure controller for a hydraulic power package (HPP) with two redundant speed variable electric motor-driven pumps (EMP) was developed. A central pressure controller, which calculates a total speed and allocates it evenly to both units, was selected. A robust control was designed applying a loopshaping method, where requirements and constraints are mapped on target loopshapes. The control design was verified in non-linear simulation and by an experiment on a dedicated test rig. It meets the specified performance requirements and offers robustness against slightly asymmetric EMP characteristics. This is an important result, as oscillations due to slightly differing pump behaviours are a well-known issue in today's aircraft multi-pump systems.

In the second part of this paper a model predictive control allocation (MPCA) was developed, to take advantage of the low utilization during most parts of the flight. The MPCA algorithm solves an optimization problem, which minimizes a cost function for a secondary objective and satisfies control limitations as constraints, to calculate the speed allocation. A first test case showed that the algorithm improves the efficiency by prefering the more efficient unit. The improvement is, however, rather low but increases with increasing differences between the units. In a second test the MPCA algorithm was demonstrated to improve the dynamic performance in case of asymmetrically degraded units by prioritizing the better one. This is a promising result to enable fault-tolerant control.

Overall, the MPCA algorithm provides the baseline for the implementation of a holistic and integrated HPP operating strategy. Future investigations need to verify the MPCA strategy on the test rig. This should also include the investigation of additional optimization objectives, e.g. the minimization of noise emissions, wear and tear effects. Furthermore, the MPCA algorithm needs to be coupled to the HUMS, which provides the essential plant information (like pump efficiencies, limits) and the potential of integrating also the pressure controller into the MPC formulation should be explored.

\section{Acknowledgement}

This work was funded by the German Federal Ministry of Economic Affairs and Energy (BMWi) within the MODULAR project (contract code: 20Y1910G) in the national LuFo VI-1 program. Their support is greatly appreciated.

The authors would further like to thank Liebherr-Aerospace Lindenberg $\mathrm{GmbH}$ for their support, provision of the prototype EMPs, and funding of the test rig. It has received funding from the Clean Sky 2 Joint Undertaking under the European Union's Horizon 2020 research and innovation programme under grant agreement No 807097.

Thanks also go to Jonathan Jobmann, who made an important contribution to the conceptualization of the basic control structure as part of his master's thesis. Last but not least, the authors would like to thank Liesa Voges, whose work provided an important basis for the MPCA concept development. 


\section{References}

[1] R. I. Jones. The more electric aircraft - assessing the benefits. Proceedings of the Institution of Mechanical Engineers, Part G: Journal of Aerospace Engineering, 2002.

[2] N. Trochelmann, T. Rave, F. Thielecke, and D. Metzler. An investigation of electro-hydraulic high efficient power package configurations for a more electric aircraft system architecture. In Deutscher Luft-und Raumfahrtkongress, Munich, 2017.

[3] SAE International (Publ.). Aerospace Information Report (AIR) 5005 - Commercial Aircraft Hydraulic Systems. Society of Automotive Engineers (SAE), 2015.

[4] Boeing (Publ.). 787 No-Bleed Systems: Saving Fuel and Enhancing Operational Efficiencies. AERO magazine, 2007.

[5] Boeing. Dynamic activation of pumps of a fluid power system, 2018.

[6] SAE International (Publ.). Aerospace Information Report (AIR) 4553B - Aerospace Hydraulics and Actuation Lessons Learned. Society of Automotive Engineers (SAE), 2010.

[7] S. Guo, J. Chen, Y. Lu, Y. Wang, and H. Dong. Hydraulic piston pump in civil aircraft: Current status, future directions and critical technologies. Chinese Journal of Aeronautics (CJA), 2019.

[8] J. E. Spencer. Development of Variable Pressuere Hydraulic Systems for Military Aicraft Utilizing the Smart Hydraulic Pump. In ImechE, 1993.

[9] W. Davidson and J. Roizes. Electro-Hydrostatic Actuation System for Aircraft Landing Gear Actuation. In Workshop on Aircraft System Technologies, 2015.

[10] N. Elliott, S. Linforth, and C. Moore. Thermae ii (main landing gear \& door eh actuation system) integration and testing. In Recent Advances in Aerospace Actuation Systems and Components, March 16-18, 2016, Toulouse, France, 2016.

[11] A. Banaszek and R. Petrovic. Problem of non proportional flow of hydraulic pumps working with constant pressure regulators in big power multipump power pack unit in open system. In Technical Gazette 26, 2(2019), 294-301, 2019.

[12] I. Rühlicke. Elektrohydraulische Antriebssysteme mit drehzahlveränderbarer Pumpe. PhD thesis, Technische Universität Dresden, Institut für Fluidtechnik, 1997.

[13] T. Neubert. Untersuchungen von drehzahlveränderbaren Pumpen. PhD thesis, TU Dresden, 2002.

[14] A. Helbig. Energieeffizientes elektrisch-hydrostatisches Antriebssystem am Beispiel der KunststoffSpritzgiessmaschine. PhD thesis, TU Dresden, 2007.

[15] K. Ritters, L. Roos L., and L. Frerichs. Efficiency Studies on Double Pump Supply Units. In 11th International Fluid Power Conference, Aachen, 2018.

[16] L. Roos, J. Untch, and L. Frerichs. Effizienzsteigerungen durch 2-Pumpen-Versorgungseinheiten. In Hybride und energieeffiziente Antriebe für mobile Arbeitsmaschinen, 2015.

[17] J. Viholainen, J. Tamminen, T. Ahonen, J. Ahola, E. Vakkilainen, and R. Soukka. Energy efficient control strategy for variable-speed driven parallel pumping systems. In Springer Energy Efficiency Journal, 2013.

[18] N. Trochelmann, P. Bischof, F. Thielecke, D. Metzler, and S. Bassett. A robust pressure controller for a variable speed ac motor pump - application to aircraft hydraulic power packages. In Bath/ASME 2018 Symposium on Fluid Power and Motion Control (FPMC), 2018.

[19] R. Krishnan. Permanent Magnet Synchronous and Brushless DC Motor Drives. Taylor and Francis Group, LLC, 2010.

[20] D. Schroeder. ElektrischeAntriebe - Regelung von Antriebssystemen. Springer-Verlag Berlin Heidelberg, 3 edition, 2009.

[21] SAE International (Publ.). Aerospace Standard (AS) 595 - Pressure Compensated variable displacement aircraft pumps - Revision D. 2010. 
[22] M. Franke. Intelligente Antriebe im Systemverbund - Ein Beitrag zur Untersuchung des Betriebsverhaltens von dezentralen feldbusgekoppelten Antrieben. PhD thesis, TU Dresden, 1999.

[23] H. Niemann. Synchronisierte Regelung dezentraler Antriebe über einen Feldbus. PhD thesis, University Erlangen-Nürnberg, 2001.

[24] H. Geilsdorf. Modellbasierter Entwurf der Synchronisationsregelung und Fehlerüberwachung elektrischer Einzelantriebe in Landeklappensystemen. PhD thesis, TUHH, 2009.

[25] S. Skogestad and I. Postlethwaite. Multivariable Feedback Control. Prentice Hall (ACHTUNG: TBD), 2005.

[26] T. A. Johannsen and Thor I. Fossen. Control allocation - a survey. In Automatica, volume 49, pages 10871103, 2013.

[27] C. Satzger, R. d. Castro, and T. Bünte. A model predictive control allocation approach to hybrid braking of electric vehicles. In IEEE Intelligent Vehicles Symposium, 2014.

[28] M. Hanger, T. A. Johannsen, G. K. Mykland, and A. Skullestad. Dynamic model predictive control allocation using cvxgen. In 9th IEEE International Conference on Control and Automation (ICAA), 2011.

[29] J. Willkomm. Modellprädiktive Optimierung drehzahlvariabler Verstellpumpen. PhD thesis, Technical University of Darmstadt, 2016.

[30] F. Bakshande, M. Spiller, Y.-L. King, and D. Söffker. Computationally efficient model predictive control for real time implementation experimentally applied on a hydraulic differential cylinder. In 21st IFAC World Congress, 2020.

[31] L. Wang. Model Predictive Control System Design and Implementation Using MATLAB. Springer-Verlag Londong Limited, 2009. 